\title{
Apoyos para el aprendizaje de las matemáticas según apreciación de los estudiantes adolescentes
}

\author{
Supports for learning mathematics according to the appreciation of teenage \\ students
}

SALINAS-AGUIRRE, María del Consuelo†*, HERNÁNDEZ-CUETO, Jaquelina Lizet; YAÑEZFLORES, Sara Margarita y SOLIS-PERALES, Sergio Arturo

Universidad Autónoma de Coahuila, Departamento de Postgrado e Investigación de la Facultad de Ciencia, Educación y Humanidades, Edificio "N" Unidad Camporredondo, Saltillo Coahuila, México.

ID $1^{\text {er }}$ Autor: María del Consuelo, Salinas-Aguirre / ORC ID: 0000-0002-6542-1813, Researcher ID Thomson: S-92442018, CVU CONACYT ID: 615335

ID $1^{\text {er }}$ Coautor: Jaquelina Lizet, Hernández-Cueto / ORC ID: 0000-0002- 3728- 7434, Researcher ID Thomson: S-85882018, CVU CONACYT ID: 322702

ID $2^{\text {do }}$ Coautor: Sara Margarita, Yañez-Flores / ORC ID: 0000-0002-4750-4244, Researcher ID Thomson: S-9231-2018, CVU CONACYT ID: 352125

ID $3^{\text {er }}$ Coautor: Sergio Arturo, Solis-Perales / CVU CONACYT ID: 1013578

DOI: $10.35429 / J P D .2019 .9 .3 .19 .29$

Recibido 10 de Agosto, 2019; Aceptado 30 de Septiembre, 2019

\begin{abstract}
Resumen
Esta investigación identifica atributos del aprendizaje matemático del estilo de vida de bachilleres, habilidades docentes y factores personales, escolares y familiares que potencializan habilidades numéricas. El diseño es no experimental cuantitativo, de corte transversal aplicado a una muestra aleatoria de 423 estudiantes con un instrumento validado (Cronbach 0.94). Tiene 14 variables signalíticas y 192 variables simples con tres ejes: aprendizaje matemático, habilidades docentes y estilo de vida, doce variables complejas: conductas, actitudes y emociones estudiantiles, en clase, con tareas, percepción matemática, manejo tiempos y autocuidado. Aspectos familiares-sociales. Evaluaciones y estrategias didáctico-matéticas y emocionales respecto al maestro. Tecnologías para matemáticas. El análisis descriptivo, correlacional y multifactorial, es realizado con porcentajes y variabilidad, en correlacional con Pearson y por Comunalidades $(\mathrm{p}=0.5)$. Los resultados muestran importantes son: hábitos y tareas, actividades y didáctica, organización familiar y relaciones afectivas que intervienen en aprendizajes personales. Destacan variables subjetivas y actitudinales hacia estudiar, matemáticas y entorno familiar que potencializa o no el aprendizaje Las contribuciones son: metodologías didáctico-matéticas de matemáticas y un modelo flexible multifactorial de aprendizajes matemáticos acorde a personalidades y distintas formas de aprender de los estudiantes, que considera: percepción y actitudes hacia abstracciones numéricas, habilidades cognitivas, acciones áulicas, didáctica docente y contexto social.
\end{abstract}

Metodología, Aprendizaje, Matemáticas

\begin{abstract}
This research identifies attributes of mathematical learning in the lifestyle of high school students, teaching skills and personal, school and family factors that potentiate numerical skills. The design is nonexperimental quantitative, cross-sectional applied to a random sample of 423 students with a validated instrument (Cronbach 0.94). It has 14 signaling variables and 192 simple variables with three axes: mathematical learning, teaching skills and lifestyle, twelve complex variables: student behaviors, attitudes and emotions, in class, with homework, mathematical perception, time management and healthcares. Family-social aspects. Didactic-subjects and emotional evaluations and strategies regarding the teacher. Technologies for mathematics. The descriptive, correlational and multifactorial analysis is carried out with percentages and variability, in correlation with Pearson and by Communalities $(\mathrm{p}=0.5)$. The results show important are: habits and tasks, activities and didactics, family organization and emotional relationships that intervene in personal learning. Highlights subjective and attitudinal variables towards studying, mathematics and family environment that potentiates learning or not. The contributions are: didactic-subject's methodologies of mathematics and one flexible multifactorial model of mathematical learning according to personalities and different ways of learning from students, which considers: perception and attitudes towards numerical abstractions, cognitive skills, classroom actions, teaching didactics and Social context.
\end{abstract}

Keywords. Methodology, learning, mathematics

Citación: SALINAS-AGUIRRE, María del Consuelo†*, HERNÁNDEZ-CUETO, Jaquelina Lizet; YAÑEZ-FLORES, Sara Margarita y SOLIS-PERALES, Sergio Arturo. Apoyos para el aprendizaje de las matemáticas según apreciación de los estudiantes adolescentes. Revista de Didáctica Práctica. 2019. 3-9: 19-29.

\footnotetext{
*Correspondencia al Autor (Correo Electrónico: consuelo.salinas@uadec.edu.mx)

$\dagger$ Investigador contribuyendo como primer autor.
} 


\section{Introducción}

El aprendizaje de las matemáticas a la par con la lectoescritura es la esencia de la educación formal y desarrollo de los estudiantes para la vida futura.

La presente investigación tiene como objetivo encontrar los atributos que están presentes en el estilo de vida del estudiante, las habilidades del docente y procesos didácticos que contribuyen al aprendizaje matemático en educación media superior. La tesis central de la investigación es conocer los atributos están presentes en un aprendizaje efectivo de las matemáticas. Los temas de la investigación refieren a factores del estilo de los estudiantes de preparatoria, a conductas escolares y externas entre ellas relación parental y con amigos; por otra parte las habilidades docentes y la didáctica que aplican los maestros y el uso de tecnologías de punta que inciden en el aprendizaje matemático de los adolescentes que cursan el nivel de la preparación formal en el nivel secundaria de la educación formal.

\section{Antecedentes}

Son muy variadas las concepciones de aprendizaje Abbagnano N. (1974) en el Diccionario de Filosofía define al aprendizaje como "la adquisición de técnicas simbólicas, emotivas o de comportamiento", es decir, un cambio de las conductas personales al ambiente que mejora las respuestas hacia fines filogenéticos de adaptación, conservación y desarrollo de un organismo vivo.

Platón es el primero en ilustrar esta noción en su "teoría de la anamnesis", la cual estipula, del aprendizaje como la asociación de cosas o ideas entre sí, que permiten al alma el aprehender, que significa ligar o relacionar ideas, así los humanos se apropian del saber en el conocer del sujeto mismo.

El iniciador de la pedagogía científica y experimental, Herbart J. F. (1806), “Ciencias de la educación", afirma del aprendizaje como una “apercepción”, fenómeno por el cual una masa de representaciones integra en sí, una nueva representación, que puede conectarse con las demás de algún modo, actualmente estudiado en las Neurociencias.
Thorndike E. L. psicólogo conductista ilustra el concepto de aprendizaje definiéndolo, como un proceso de tanteo guiado por la respuesta dada y respondida en el ambiente con premio ò castigo, ante una necesidad un estímulo condicionado puede quedar unido o ligado a una respuesta que lo sigue según el reforzador externo que le precede, lo que el sujeto aprehende es la relación entre signos y respuestas, constituyendo un constructo de experiencias de aprendizajes.

El físico Resnick R. (1989) asegura del aprendizaje de las matemáticas, que "la mayoría de las investigaciones del aprendizaje de los números y la aritmética constituyen una parte importante del currículum escolar y los conceptos numéricos que representan la base sobre la cual, se pueden desarrollar elevadas competencias numéricas", así en un ambiente natural la cognición humana construye por si misma conceptos o constructos matemáticos para la sobrevivencia.

Piaget J, (1958) desarrolla la teoría del aprendizaje por etapas con los procesos de asimilación y acomodación hasta llegar al pensamiento lógico abstracto/matemático en la adolescencia. Vygotsky L. (1978) trata el desarrollo cognitivo en la mediación personal y simbólica, "los niños empiezan a estudiar aritmética en la escuela, pero mucho tiempo antes han tenido ya alguna experiencia con ella". Bermejo R. (1990) "los niños construyen la aritmética en dos contextos separados: en el aula y fuera de ella", (2016) "los niños experimentan y manipulan por placer", así es como aprenden y la cognición se constituye. Brissiaud R. (1993) matemático y doctor en psicología cognitiva dice "los niños saben calcular más o menos, pero no saben resolver problemas".

En la formación de actitudes positivas hacia el estudio de la ciencia, Ortega P. Saura J. P. y Mínguez R. aseguran que las actitudes tienen tres componentes: cognitivo, afectivo y la intención conductual de la acción, el aspecto emocional tiene mayor participación en una actitud que es aprendida y se asocia al contexto y situación relacionada, pero respecto a la ciencia, las actitudes incluyen rasgos como curiosidad, objetividad y orientación cognitiva. 


\section{Metodología}

La investigación es de tipo exploratorio, no experimental de corte transversal. Tiene tres ejes: $1^{\circ}$. Conductas ante las matemáticas; $2^{\circ}$. Estilos de vida de los estudiantes; $3^{\circ}$. Habilidades y didáctica de los docentes. Las variables complejas del estudiante son: acciones ante dudas de clase, conductas del salón de clase, acciones de organización familiar y relaciones parentales, emociones y actitudes aprendidas ante las matemáticas, manejo del tiempo libre, alimentación, relaciones con iguales. En las variables complejas del maestro se miden: estrategias didácticas, manejo de tecnología para clase y valores. El instrumento de medición es un cuestionario electrónico con 192 variables simples en una escala de medición decimal y 16 variables signalíticas para la caracterización de la muestra estudiantil realizada con 423 sujetos de una población de 3760 estudiantes del nivel medio superior seleccionados al azar en cuatro planteles de una universidad privada de nivel socio-económico medio alto. Se realza una prueba piloto del instrumento electrónico con 25 sujetos y se valida con un Alpha de Cronbach de 0.94. Después de recolectar los datos en cohorte horizontal y vía plataforma universitaria virtual, para dar explicación a la investigación, se realiza un análisis descriptivo, uno correlacional por ejes y uno factorial con el método por Comunalidades para realizar inferencias predictivas de datos.

Para caracterizar la muestra de estudiantes que inciden en conductas que ayudan al aprendizaje de las matemáticas y por otro lado, las acciones y didáctica potencializadoras de los maestros de matemáticas y demás relaciones externas con amigos y padres, se realiza un análisis descriptivo de datos en lecturas de frecuencias y porcentajes y análisis estadístico de medidas de tendencia central y variabilidad.

Para conocer las características del fenómeno son analizadas variables ordinales con el estadígrafo de correlación con Pearson y también es incluido un análisis de factores multivariado por comunalidades con un nivel de probabilidad de error menor a 0.05 en las variables de los tres ejes y 14 variables complejas de investigación.

\section{Resultados}

Se realiza un análisis en tres etapas: descriptivo, correlacional y multivariado.

\section{Análisis descriptivo}

La muestra de estudiantes tiene las siguientes características: las modalidades curriculares de estudios pertenecen a estratos bilingüe, multicultural e internacional. El $35 \%$ de los respondientes pertenecen al primer grado, inscritos en la modalidad multicultural; $55 \%$ de la muestra son mujeres, con edades que oscilan entre 14 y 18 años, con un promedio 17 años; el $80 \%$ de los padres tienen un estado civil casados; la cantidad de hermanos está en un rango de entre 1 y 5 hermanos la media es de 2 hermanos; el $51 \%$ de los estudiantes vive con ambos padres; el 53\% de ambos padres tiene una carrera universitaria; el $97 \%$ de los padres trabaja y el $52 \%$ tiene negocio propio; de las madres el $55 \%$ trabaja y de ellas el $31 \%$ es empleada.

El análisis de las medidas de tendencia central, tienen resultados altos en el eje de las conductas que realiza el estudiante cuando tiene dudas ellos dicen acuden al maestro, amigo o compañero o investiga por sí solos, no asisten a asesorías, con el tutor, ni se queda con las dudas matemáticas. El estudiante acepta que necesita ayuda de otra persona como amigos, maestros o tutores. En cuanto a la clase de matemáticas, los estudiantes disfrutan cómo el maestro imparte la clase, la comprenden, les gusta y les hace sentir seguros, por lo que el maestro es una figura importante en el aprendizaje. En cuanto a factores áulicos, los valores altos para aprender matemáticas son las variables: poner atención y comprender instrucciones, pensar y analizar cómo resolver y evaluar opciones, la ayuda de compañeros y participación en grupo son importantes. Lo que no le ayuda a aprender es: utilizar agenda, revisar el programa, hacer tarea en otra clase, estar al pendiente de alguna red social durante la clase matemática o salirse de la clase, esto indica falta de organización de los estudiantes y son sujetos a distraerse con aparatos de alta tecnología. Las conductas de casa que favorecen el aprendizaje de matemáticas son: tener ordenadas las cosas de la escuela, reconocer ser el responsable del aprendizaje, utilizar más de dos horas las redes sociales en los problemas a resolver. 
El eje estilo de vida fue medido a través del manejo del tiempo libre y la autopercepción con respecto a acciones que realizan los estudiantes fuera de la escuela. En el análisis de medias de medias los sujetos indican que suelen estar de buen humor, satisfechos con la vida, hacen ejercicio, se sienten a gusto con el cuerpo, ser sedentario, tener un lugar fijo para hacer las tareas, invertir el tiempo necesario hasta entender el tema y dan prioridad a la tarea de matemáticas. Todas estas conductas son propias de la adolescencia. Respecto a los hábitos alimenticios suelen ingerir agua, carne blanca, frutas, carne roja, verduras, van desayunado a la escuela y comen rápido. Los puntajes bajos están en las variables: comer comida rápida, durar más de 6 horas sin consumir alimentos, tomar refresco, saltarse las comidas, consumir café, suplementos alimenticios ò tomar algo para adelgazar. Estos atributos positivos indican tienen una buena alimentación, posiblemente por el estrato económico medio/alto y los padres con alto nivel académico. En cuanto a la relación que mantienen con iguales: tienen por lo menos dos amigos, platican físicamente con ellos, escuchan confidencias, dicen sentirse aceptados, platican con medios electrónicos, se juntan por tener los mismos gustos, van a fiestas, suelen visitarse en los cumpleaños. Los amigos son parte indispensable de la autoestima $\mathrm{y}$ vida de los adolescentes.

Suelen sentirse bien con el padre de familia, les da dinero, les preguntan cómo le ha ido, se encarga de la alimentación, el papá se lleva bien con la esposa, dan confianza y reconocen los padres el esfuerzo que hace el hijo. Y son variables respecto a padres: pocos asisten a juntas de la escuela (ellos trabajan), establecen un horario para dormir y para hacer las tareas.

Las madres son las que ponen las reglas de disciplina en casa y tienen tiempo de ir a juntas. Ellas se encargan de la alimentación y pendientes escolares, estos hijos lo reconocen y tienen una buena relación con las madres. La falta de límites en horario de dormir y hacer tarea es un factor que influye en el proceso de aprendizaje, puesto que los padres de familia no establecen un horario para hacer la tarea, por lo que faltan hábitos y organización en la familia, esto se debe posiblemente a que la mitad de las madres trabajan también como los padres.
En cuanto al comportamiento de los maestros podemos notar como positivos cuando el maestro utiliza software para ejercicios, recurre al Blackboard (plataforma educativa electrónica de la universidad), pone proyectos en línea y presentaciones en Power point, también gustan de evaluaciones en línea y en menor proporción tutoriales en Youtube, foros de discusión y software para graficar. El uso de los recursos tecnológicos favorece el proceso de aprendizaje matemático. El maestro mide el aprendizaje utilizando: evaluación individual, ejercicios de práctica, aprender de los errores y corregirlos y evaluar al compañero.

Las variables positivas que integran el fenómeno de estudio en general las presentan un alto grado de predictibilidad, lo muestra la lectura " $z$ " de por lo que pueden ser extrapoladas a otras poblaciones similares. Además, el coeficiente de variación muestra en todos los ejes homogeneidad de datos en las respuestas.

\begin{tabular}{|c|c|c|c|c|c|c|c|}
\hline Variable & $n$ & $\bar{x}$ & s & Sk & $\mathrm{K}$ & $\mathrm{cV}$ & $\mathrm{z}$ \\
\hline 13 Pensar cómo resolver & 423 & 8.24 & 1.97 & -1.55 & 3.22 & 23.96 & 4.17 \\
\hline 1 Maestro & 423 & 7.85 & 2.28 & -1.20 & 1.17 & 29.04 & 3.44 \\
\hline 19 Analizar los ejercicios & 423 & 7.68 & 2.24 & -1.14 & 1.17 & 29.10 & 3.44 \\
\hline 21 Comprende instrucciones & 423 & 7.68 & 2.15 & -0.92 & 0.54 & 27.98 & 3.57 \\
\hline 25 Participar activamente en equipo & 423 & 7.55 & 2.59 & -1.20 & 0.96 & 34.24 & 2.92 \\
\hline 27 Ayudar a compañeros & 423 & 7.47 & 2.90 & -1.18 & 0.49 & 38.80 & 2.58 \\
\hline 35 Evaluar opciones & 422 & 7.44 & 2.42 & -1.04 & 0.74 & 32.51 & 3.08 \\
\hline 93 Motiva a participar (el profesor) & 423 & 7.26 & 3.17 & -1.05 & -0.05 & 43.59 & 2.29 \\
\hline 67 Reconoce que es responable el aprendizaje & 423 & 7.24 & 2.87 & -0.93 & -0.05 & 39.69 & 2.52 \\
\hline 57 Invertir tiempo hasta entender & 423 & 7.03 & 2.62 & -0.77 & -0.12 & 37.26 & 2.68 \\
\hline 30 Proponer soluciones & 423 & 6.99 & 2.90 & -0.88 & -0.12 & 41.53 & 2.41 \\
\hline 47 Disfurta cómo imparte el profesor & 423 & 6.85 & 3.02 & -0.83 & -0.23 & 44.08 & 2.27 \\
\hline 46 La entiende & 423 & 6.55 & 2.91 & -0.82 & -0.13 & 44.37 & 2.25 \\
\hline 37 Le gusta & 423 & 6.15 & 3.51 & -0.56 & -1.06 & 57.01 & 1.75 \\
\hline 39 Lo pone nervioso & 423 & 6.02 & 3.54 & -0.45 & -1.20 & 58.74 & 1.70 \\
\hline 38 Lo hace sentir seguro & 423 & 5.67 & 3.54 & -0.38 & -1.19 & 62.50 & 1.60 \\
\hline 49 Realizar ejercicios en casa & 423 & 5.57 & 3.18 & -0.32 & -1.02 & 57.05 & 1.75 \\
\hline 44 Cuesta trabajo entenerla & 423 & 5.54 & 3.43 & -0.25 & -1.21 & 61.95 & 1.61 \\
\hline 41 Se aplica ala vida diaria & 423 & 5.49 & 3.51 & -0.19 & -1.31 & 63.88 & 1.57 \\
\hline 40 Es fácil & 423 & 5.43 & 3.38 & -0.29 & -1.18 & 62.21 & 1.61 \\
\hline 14 Esperar que el profesor resuelva & 423 & 5.42 & 2.74 & -0.21 & -0.73 & 50.61 & 1.98 \\
\hline 60 Tener ay uda financiera beca & 423 & 5.35 & 4.63 & -0.17 & -1.86 & 86.63 & 1.15 \\
\hline 48 Es divertida & 423 & 5.09 & 3.40 & -0.09 & -1.26 & 66.87 & 1.50 \\
\hline 43 Es aburrida & 423 & 4.33 & 3.25 & 0.27 & -1.02 & 75.12 & 1.33 \\
\hline 7 Asistir a asesorias & 423 & 4.30 & 3.54 & 0.18 & -1.36 & 82.12 & 1.22 \\
\hline 45 Lo derrota & 423 & 3.78 & 3.63 & 0.50 & -1.20 & 96.13 & 1.04 \\
\hline 8 Le es indiferente & 421 & 3.30 & 3.18 & 0.59 & -0.76 & 96.53 & 1.04 \\
\hline 2 Tutor externo & 423 & 3.30 & 3.74 & 0.59 & -1.26 & 113.50 & 0.88 \\
\hline 6 Quedarse con la duda & 423 & 3.28 & 3.09 & 0.63 & -0.68 & 94.13 & 1.06 \\
\hline 15 Pasar al pizarrón & 423 & 3.19 & 3.01 & 0.67 & -0.53 & 94.30 & 1.06 \\
\hline $\bar{X} \bar{x}=5.90 \quad$ Ss $=0.56 \quad L i=5.34$ & & & & & & & \\
\hline
\end{tabular}

Tabla 1 Análisis de medidas de tendencia central y variabilidad Percepción hacia la clase de matemáticas Fuente: elaboración propia

\section{Análisis Correlacional}

De las variables complejas en correlaciones realizadas con el Momento Producto de Pearson con un nivel probable de error de $\mathrm{p} \leq 0.001 \mathrm{y} \mathrm{r}^{2}$ $=0.159$. 
En los resultados podemos notar que los estudiantes para aprender utilizan habilidades cognitivas, realiza acciones en el salón para aprender; reconocen al profesor como importante para acarar dudas. Los procesos cognitivos de cómo aprende y comprende potencializan las conductas de gestión para el aprendizaje matemático. También podemos notar que al no utilizar actitudes positivas y habilidades cognitivas no logran aclarar las dudas de la materia.

Las matemáticas están relacionadas con la afectividad, lo que puede facilitar o dificultar que el aprendizaje.

Las habilidades del docente potencializan el tipo de percepción matemática, que ayudará al estudiante cuando es positiva, demás influye la forma de evaluación para que el alumno utilice sus insumos cognitivos. Es importante destacar que no es relevante dónde se siente los estudiantes dentro del aula, ni la forma en que son resueltos los problemas matemáticos, ya sea de forma individual o por pares, sino lo significativo es que puedan aplicar el conocimiento matemático a la vida diaria.

Cuando los estudiantes se sienten nerviosos, aburridos, derrotados, les cuesta trabajo entender matemáticas y le es indiferente que el profesor sea organizado, muestre la planeación del curso, y diga lo que se espera aprender en la clase. La planeación, didáctica y las estrategias de enseñanza aprendizaje realizadas por el maestro ayudan al estudiante a desarrollar los procesos cognitivos y a responsabilizarse del propio aprendizaje.

Los estudiantes no utilizan las redes sociales con fines estudio, lo hacen para fortalecer la afectividad y estar en comunicación con sus iguales. Además, los aprendizajes deben complementarse con aulas invertidas, abiertas aprovechando la vinculación de actividades externas, para aprendizajes en las instituciones productivas. Cuando los estudiantes tienen dudas matemáticas acude con el profesor y realizan acciones para resolverlas en el salón de clase como: pasar al pizarrón, comprende las instrucciones, analiza el ejercicio, piensan cómo resolverlo, evaluar opciones, propone soluciones y ayuda a los compañeros y participan en equipos de trabajo.
En síntesis, las acciones que realizan los estudiantes para comprender las matemáticas se asocian con el aprendizaje apoyado en las habilidades del docente, la percepción positiva, el uso de habilidades cognitivas, las acciones realizadas en casa.

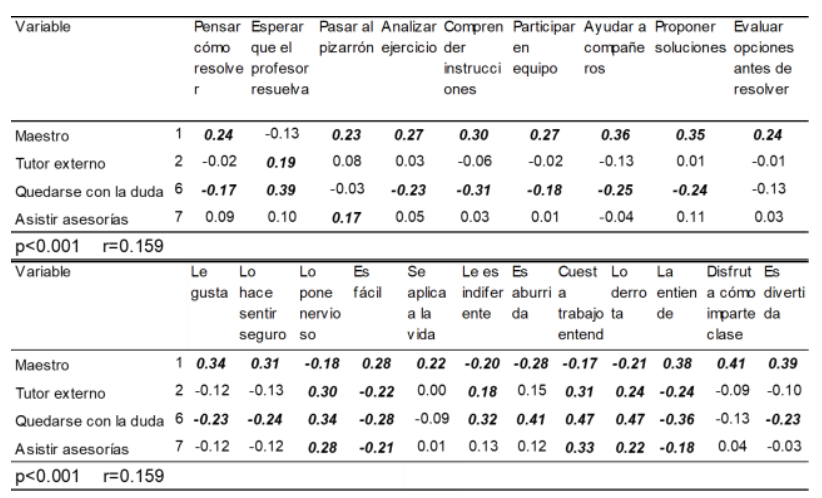

Tabla 2 Análisis correlacional de manejo de la duda: acciones áulicas, percepción matemática y habilidad del docente

Fuente: elaboración propia

\begin{tabular}{|c|c|c|c|c|c|c|c|c|c|c|c|c|}
\hline & \multirow{2}{*}{\multicolumn{2}{|c|}{ Motiva Tono }} & \multirow[b]{2}{*}{$\begin{array}{l}\text { Orga } \\
\text { niza }\end{array}$} & \multirow[b]{2}{*}{$\begin{array}{l}\text { Plan } \\
\text { eac }\end{array}$} & \multirow[b]{2}{*}{$\begin{array}{l}\text { Nom } \\
\text { bre }\end{array}$} & \multirow[b]{2}{*}{$\begin{array}{l}\text { Esp } \\
\text { era }\end{array}$} & \multicolumn{3}{|c|}{ Contr } & \multirow[b]{2}{*}{$\begin{array}{l}\text { Sabe } \\
\text { tem }\end{array}$} & \multirow[b]{2}{*}{$\begin{array}{l}\text { Salu } \\
\text { da }\end{array}$} & \multirow[b]{2}{*}{$\begin{array}{l}\text { Paci } \\
\text { ente }\end{array}$} \\
\hline & & & & & & & $\begin{array}{l}\text { Atent } \\
0\end{array}$ & $\begin{array}{l}\text { aeje } \\
\mathrm{m}\end{array}$ & $\begin{array}{l}\text { Expli } \\
\text { ca }\end{array}$ & & & \\
\hline Le gusta & 0.08 & 0.07 & -0.02 & 0.03 & 0.07 & -0.04 & 0.01 & 0.00 & 0.01 & 0.04 & 0.04 & 0.07 \\
\hline Seguro & 0.10 & 0.08 & 0.02 & 0.06 & 0.08 & -0.06 & 0.03 & -0.01 & 0.01 & 0.07 & 0.06 & 0.06 \\
\hline Nervioso & -0.04 & -0.01 & 0.08 & 0.07 & 0.04 & 0.13 & 0.02 & 0.10 & 0.04 & -0.01 & 0.00 & -0.02 \\
\hline Es fácil & 0.13 & 0.03 & -0.03 & 0.03 & 0.05 & -0.06 & 0.00 & -0.01 & -0.01 & 0.04 & 0.04 & 0.08 \\
\hline Aplica vida & 0.06 & 0.06 & -0.02 & 0.04 & 0.05 & 0.00 & 0.03 & 0.01 & 0.03 & 0.01 & 0.00 & 0.05 \\
\hline Indiferente & -0.02 & -0.04 & 0.12 & 0.09 & 0.09 & 0.13 & 0.01 & 0.13 & 0.01 & -0.04 & -0.04 & -0.08 \\
\hline Aburrida & -0.02 & 0.02 & 0.13 & 0.10 & 0.14 & 0.18 & -0.01 & 0.14 & 0.00 & -0.01 & -0.05 & -0.06 \\
\hline Trab enten & -0.05 & 0.01 & 0.09 & 0.07 & 0.07 & 0.17 & 0.00 & 0.13 & 0.04 & -0.01 & -0.03 & -0.05 \\
\hline Lo derrota & -0.03 & -0.01 & 0.10 & 0.09 & 0.08 & 0.19 & 0.00 & 0.18 & 0.01 & -0.04 & -0.07 & -0.11 \\
\hline La entiende & 0.14 & 0.09 & 0.01 & 0.04 & 0.05 & -0.03 & 0.07 & -0.01 & 0.05 & 0.11 & 0.09 & 0.11 \\
\hline Disfruta pro & 0.05 & 0.10 & 0.05 & 0.05 & 0.03 & -0.02 & 0.12 & -0.04 & 0.09 & 0.14 & 0.11 & 0.07 \\
\hline Divertida & 0.08 & 0.09 & 0.01 & 0.05 & 0.07 & -0.02 & 0.08 & -0.01 & 0.06 & 0.10 & 0.07 & 0.06 \\
\hline$\leq 0.0$ & & & & & & & & & & & & \\
\hline
\end{tabular}

Tabla 3 Análisis correlacional de percepción matemática y acciones que realiza el docente en clase

Fuente: elaboración propia

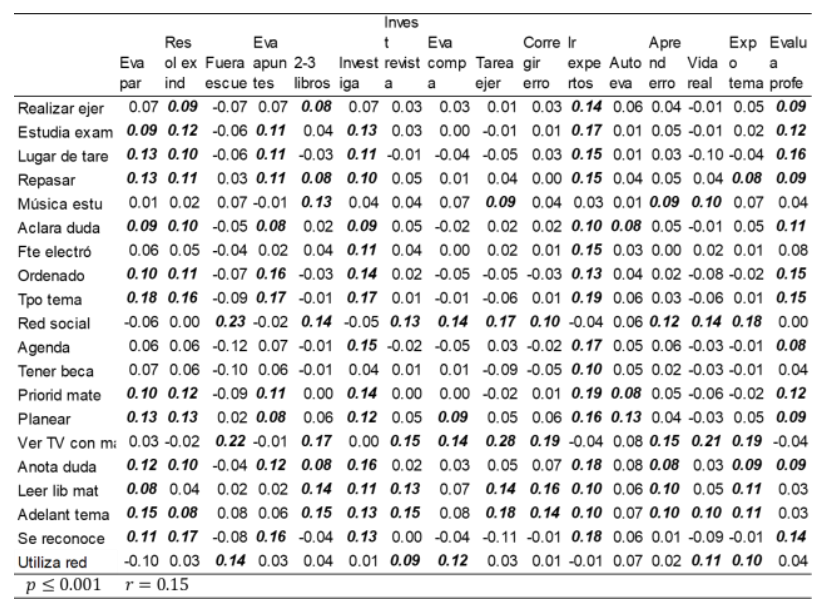

Tabla 4 Análisis correlacional de acciones que realiza el estudiante en casa y la forma en que el docente mide el conocimiento

Fuente: elaboración propia 


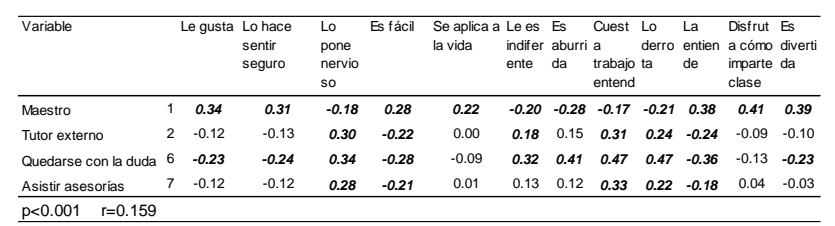

Tabla 5 Análisis correlacional del manejo de la duda con relación a la percepción matemática

Fuente: elaboración propia

\section{Análisis Factorial}

Se realizó un análisis de Factores Multivariado por Comunalidades con rotación varimax normalizada, con el criterio de Káiser y una $\mathrm{r}^{2}$ múltiple, error probable $\leq 0.001$ y nivel de confianza del $99.99 \%$. Se presentan 20 factores que explican el $43.34 \%$ de la variabilidad del fenómeno estudiado con un $\mathrm{r}=\geq 0.159$. Se analiza con un corte signalítico en las lecturas hasta el cuarto factor, porque son los que refieren al $28.21 \%$ de explicación del total.

\begin{tabular}{|c|c|c|c|c|}
\hline 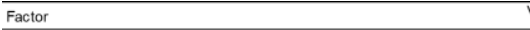 & 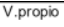 & Total $S$ & V.prop & \\
\hline 1. La enseñanza en el aprendizaje matemático & 25.43 & 13.25 & 25.43 & 13.25 \\
\hline 2. Medición del aprendizaje acciones para aprender & 12.39 & 6.45 & 37.82 & 19.70 \\
\hline 3. Alimentación saludable y contexto farmiliar como gestores del aprendizaje & 10.23 & 5.33 & 48.05 & 25.03 \\
\hline 4. Locus de control del aprendizaje matemático & 6.10 & 3.18 & 54.15 & 28.21 \\
\hline 5. Autopercepción acorde al rol dela figura paterna como base del aprendizaje & 4.42 & 2.30 & 58.57 & 30.51 \\
\hline 6. Percepción matemática acorde a acciones del estudiante & 3.79 & 1.97 & 62.36 & 32.48 \\
\hline 7. Aprendazaje como apoyo de los iguales & 2.87 & 1.50 & 65.23 & 33.98 \\
\hline 8. Aprendizaje acorde al estilo de vida saludable y autopercepción & 2.10 & 1.09 & 67.33 & 35.07 \\
\hline 9. Atributos externos que se asocian al aprendizaje & 1.87 & 0.97 & 69.20 & 36.04 \\
\hline 10. Atributos del estilo de vida & 1.85 & 0.96 & 71.05 & 37.00 \\
\hline 11. Los anigos apoyo del aprendizaje & 1.54 & 0.80 & 72.59 & 37.80 \\
\hline 12. Aprendizaje acorde al reglamento familiar & 1.43 & 0.75 & 74.02 & 38.55 \\
\hline 13. Organización familiar & 1.37 & 0.71 & 75.39 & 39.26 \\
\hline 14. Atributos negativos propios de la adolescencia & 1.26 & 0.65 & 76.65 & 39.91 \\
\hline 15. Acciones de casa para aprender & 1.23 & 0.64 & 77.88 & 40.55 \\
\hline 16. Creatividad docente & 1.19 & 0.62 & 79.07 & 41.17 \\
\hline 17. Didáctica docente acorde al anbient familiar & 1.11 & 0.58 & 67.13 & 41.75 \\
\hline 18. Atributos que dificultan el aprendizaje & 1.05 & 0.54 & 68.18 & 42.29 \\
\hline 19. Conductas negativas que dificultan el aprendib & 1.02 & 0.53 & 69.20 & 42.82 \\
\hline 20. Aprendizaje en acciones escolares & 1.00 & 0.52 & 70.20 & 43.34 \\
\hline
\end{tabular}

Tabla 6 Análisis integracional, valores propios Fuente: elaboración propia

$1^{\circ}$. Factor. La enseñanza en el aprendizaje matemático. Este factor presenta mayor varianza de todos con un $25.43 \%$ de explicación del fenómeno. Aquí se reflejan las habilidades y acciones del docente dentro del aula, de tal forma que el aprendizaje de las matemáticas está en función de la enseñanza del docente, lo cual condiciona las acciones áulicas que efectúan los estudiantes; es decir, el aprendizaje matemático está centrado en las habilidades docentes que potencializa (o no), las actitudes aprendidas del estudiante hacia el aprendizaje con su connotación emocional.

\begin{tabular}{|c|c|c|c|}
\hline$\overline{E e}$ & Variable compleja & Variable simple & F1 \\
\hline \multirow[t]{5}{*}{$\begin{array}{l}\text { Aprendizaje } \\
\text { matemático }\end{array}$} & $\begin{array}{l}\text { Acciones que hace el } \\
\text { estudiante ante la duda }\end{array}$ & 1 Maestro & 0.25 \\
\hline & $\begin{array}{l}\text { Acciones que realiza el } \\
\text { estudiante en el salón de } \\
\text { clase matemática }\end{array}$ & 33 Respeto al profesor & 0.19 \\
\hline & $\begin{array}{l}\text { Percepción de la clase de } \\
\text { matemática }\end{array}$ & 47 Disfruta cómo imparte el profesor & 0.50 \\
\hline & & 48 Es divertida & 0.30 \\
\hline & $\begin{array}{l}\text { Acciones que realiza el } \\
\text { estudiante en casa con la } \\
\text { tarea matemática }\end{array}$ & 67 Reconocer es responsable del aprendizaje & 0.19 \\
\hline \multirow[t]{5}{*}{ Estilo de vida } & Relación con los amigos & 157 Platicar con ellos fisicamente & 0.21 \\
\hline & & 159 Te cuentan confidencias & 0.20 \\
\hline & & 156 Te hacen sentir aceptado & 0.19 \\
\hline & Relación con la madre de & 181 La mamá se lleva bien con su esposo & 0.19 \\
\hline & & 182 Sentirse bien cuando están juntos madre- & 0.19 \\
\hline \multirow{11}{*}{$\begin{array}{l}\text { Habilidades } \\
\text { docentes }\end{array}$} & Manejo de la tecnología (por & 69 Blackboard & 0.16 \\
\hline & parte del profesor) & 70 Proyectos en linea & 0.16 \\
\hline & Medición del conocimiento & 89 Aprender los errores & 0.40 \\
\hline & & 92 Evaluar al profesor & 0.34 \\
\hline & & 90 Aplicar el tema a la vida real & 0.28 \\
\hline & & 82 Dar ejercicios para investigar & 0.23 \\
\hline & & 86 Corregir errores del examen & 0.21 \\
\hline & & 88 Autoevaluarse & 0.19 \\
\hline & & 91 Exponer un tema de matemáticas & 0.19 \\
\hline & & 80 Tomar los apuntes como evaluación & 0.17 \\
\hline & & 85 Encargar ejercicios & 0.16 \\
\hline \multicolumn{4}{|c|}{$p=0.001 \quad r_{2} 0.159$} \\
\hline$\overline{E j}$ & Variable compleja & Variable simple & F1 \\
\hline \multirow{29}{*}{$\begin{array}{l}\text { Habilidades } \\
\text { docentes }\end{array}$} & \multirow{29}{*}{$\begin{array}{l}\text { Acciones que realiza el } \\
\text { profesor en el salón de clase } \\
\text { matemática }\end{array}$} & 110 Tener claros los objetivos & 0.70 \\
\hline & & 104 Saber explicar el tema & 0.69 \\
\hline & & 99 Ser atento & 0.69 \\
\hline & & 106 Ser paciente & 0.68 \\
\hline & & 109 Escuchar dudas & 0.67 \\
\hline & & 116 Aclarar dudas & 0.67 \\
\hline & & 102 Ser respetuoso & 0.66 \\
\hline & & 107 Dar ejemplos del tema & 0.66 \\
\hline & & 119 Dominar el contenido del curso & 0.64 \\
\hline & & 103 Explicar el ejemplo & 0.61 \\
\hline & & 115 Retroalimentación en los exámenes & 0.60 \\
\hline & & 95 Ser organizado & 0.60 \\
\hline & & 112 Estar de buen humor & 0.59 \\
\hline & & 105 Saludar antes de comenzar & 0.57 \\
\hline & & 93 Motivar a participar & 0.57 \\
\hline & & 98 Decir lo que se espera aprender & 0.56 \\
\hline & & 100 Empezar a tiempo & 0.56 \\
\hline & & 118 Terminar a tiempo & 0.55 \\
\hline & & 117 Aplicar el reglamento escolar & 0.54 \\
\hline & & 113 Dar asesorias & 0.53 \\
\hline & & 97 Saber el nombre del estudiante & 0.51 \\
\hline & & 101 Comparar ejemplo - contraejmplo & 0.51 \\
\hline & & 108 Estimular la cooperación y trabajo en equipo & 0.49 \\
\hline & & 120 Ser creativo & 0.49 \\
\hline & & 94 Uttizar cambios de voz & 0.48 \\
\hline & & 111Tomar asistencia & 0.48 \\
\hline & & 96 Mostrar la planeación & 0.42 \\
\hline & & 114 Quenta chistes en clase & 0.28 \\
\hline & & Expl.Var & 11.66 \\
\hline
\end{tabular}

Tabla 7 Análisis integracional Factor 1: La enseñanza en el aprendizaje matemático

Fuente: elaboración propia

$2^{\circ}$. Factor. Medición del aprendizaje y acciones para aprender. Tiene una variancia de $12.39 \%$ y explica el aprendizaje de las matemáticas en función de la conducta del estudiante en el salón de clase y de la evaluación docente de la materia. La didáctica del docente se planea con el objetivo de promover acciones de gestión el aprendizaje en el estudiante, pero este factor muestra como ellos se centran en la medición de conocimiento, o sea les importa la calificación más que aprender. 
El motivador en este caso es el docente con su actitudes y acciones áulicas, pero también cuenta la realización de tareas y acciones para ello en casa.

\begin{tabular}{|c|c|c|c|}
\hline Eje & Variable compleja & Variable simple & Factor 2 \\
\hline \multirow{12}{*}{$\begin{array}{l}\text { Aprendizaje } \\
\text { matemático }\end{array}$} & \multirow{8}{*}{$\begin{array}{l}\text { Acciones que hace } \\
\text { el estudiante ante la } \\
\text { duda } \\
\text { Acciones que } \\
\text { realiza el estudiante } \\
\text { en el salón de clase } \\
\text { matemática }\end{array}$} & 7 Asistir asesorías & 0.26 \\
\hline & & 2 Tutor externo & 0.17 \\
\hline & & 15 Pasar al pizarrón & 0.30 \\
\hline & & 30 Proponer soluciones & 0.27 \\
\hline & & 23 Sentarse adelante & 0.19 \\
\hline & & 31 Realizar la tarea en receso & 0.18 \\
\hline & & 25 Participar en equipo & 0.18 \\
\hline & & 33 Respeto al profesor & -0.20 \\
\hline & \multirow{4}{*}{$\begin{array}{l}\text { Acciones que } \\
\text { realiza el estudiante } \\
\text { en casa con la tarea } \\
\text { matemática }\end{array}$} & 65 Leer libros de matemáticas & 0.28 \\
\hline & & 64 Anotar las dudas & 0.20 \\
\hline & & 66 Adelantar la clase leyendo el tema & 0.19 \\
\hline & & 63 Ver televisión cuando estudias & 0.16 \\
\hline \multirow[t]{5}{*}{ Estilo de vida } & \multirow{2}{*}{$\begin{array}{l}\text { En tu alimentación } \\
\text { diaria sueles }\end{array}$} & 137 Se ingiera algo para adelgazar & 0.22 \\
\hline & & 141 Café & 0.18 \\
\hline & \multirow[t]{2}{*}{$\begin{array}{l}\text { Relación con el } \\
\text { padre de familia }\end{array}$} & $\begin{array}{l}171 \text { Papá le platica al hijo lo que le } \\
\text { sucede }\end{array}$ & 0.22 \\
\hline & & 168 Establecer horario para hacer la & 0.22 \\
\hline & $\begin{array}{l}\text { Relación con la } \\
\text { madre de familia }\end{array}$ & $\begin{array}{l}184 \text { Establecer horario para hacer la } \\
\text { tarea }\end{array}$ & 0.24 \\
\hline Eje & Variable compleja & Variable simple & Factor 2 \\
\hline \multirow{25}{*}{$\begin{array}{l}\text { Habilidades } \\
\text { docentes }\end{array}$} & \multirow{6}{*}{$\begin{array}{l}\text { Manejo de la } \\
\text { tecnología }\end{array}$} & 72 Foros de discusión & 0.39 \\
\hline & & 73 Presentaciones en Power Point & 0.27 \\
\hline & & 70 Proyectos en línea & 0.25 \\
\hline & & 71 Tutoriales en youtube & 0.21 \\
\hline & & 76 Software para graficar( Desmos) & 0.20 \\
\hline & & 74 Evaluaciones en linea & 0.18 \\
\hline & \multirow[t]{15}{*}{ Forma de evaluar } & 87 Consultar a $1 \circ 2$ expertos del tema & 0.55 \\
\hline & & 83 Investigar en $2 \circ 3$ revistas electrónicas & 0.49 \\
\hline & & 82 Dar ejercicios para investigar & 0.46 \\
\hline & & 81 Consultar dos o tres libros & 0.42 \\
\hline & & 80 Tomar los apuntes como evaluación & 0.42 \\
\hline & & 90 Aplicar el tema a la vida real & 0.41 \\
\hline & & 88 Autoevaluarse & 0.39 \\
\hline & & 92 Evaluar al profesor & 0.37 \\
\hline & & 91 Exponer un tema de matemáticas & 0.36 \\
\hline & & 79 Realizar actividades fuera de la escuela & 0.29 \\
\hline & & 86 Corregir errores del examen & 0.28 \\
\hline & & 89 Aprender de los errores & 0.24 \\
\hline & & 77 Evaluación por pares & 0.24 \\
\hline & & 84 Evaluar al compañero & 0.21 \\
\hline & & 78 Resolver examen individual & -0.18 \\
\hline & \multirow{4}{*}{$\begin{array}{l}\text { Acciones que } \\
\text { realiza el profesor en } \\
\text { el salón de clase } \\
\text { matemática }\end{array}$} & 93 Motivar a participar & 0.26 \\
\hline & & 94 Utilizar cambios de voz & 0.18 \\
\hline & & 120 Ser creativo & 0.16 \\
\hline & & Expl.Var & 4.26 \\
\hline
\end{tabular}

Tabla 7 Análisis Integracional: Factor 2: Medición del aprendizaje empoderando el rol del estudiante

$3^{\circ}$. Factor. Alimentación saludable y contexto familiar como gestores del aprendizaje. Este factor explica una varianza del $10.23 \%$ y refiere. A conductas externas saludables del estilo de vida de los estudiantes, como la alimentación balanceada y sana, no fumar ni tomar, etc. y la autopercepción física de sí mismo. También muestra el contexto familiar y su dinámica interna con los hijos. Así como también aparece de nuevo el docente con las habilidades de evaluación y uso académico de la plataforma digital de la universidad para mostrar vía digital la planeación y reforzar con actividades didácticas los ejercicios de aprendizaje matemático. También refieren los muchachos que el docente deberá tener una actitud positiva y estimulante.
Se infiere que tener un estilo de vida saludable con una alimentación sana, una autopercepción positiva para sentirse bien consigo mismo, una buena relación y comunicación con los padres de familia se asocia al aprendizaje. Una buena autopercepción acorde a la figura materna como base de aprendizaje. Y por último del maestro aparecen las actitudes, habilidades y estrategias de evaluación, uso de tecnologías y acciones didácticas que motiven el aprendizaje.

\begin{tabular}{|c|c|c|c|}
\hline Eje & Variable complejas & Variables simples & F3 \\
\hline \multirow{20}{*}{$\begin{array}{l}\text { Estilo de } \\
\text { vida }\end{array}$} & \multirow{7}{*}{$\begin{array}{l}\text { Relación con el padre } \\
\text { de familia }\end{array}$} & 166 Sentirse bien cuando están juntos padre e hijo & 0.33 \\
\hline & & 174 Dar dinero para salidas & 0.31 \\
\hline & & 176 Poner limites & 0.29 \\
\hline & & 175 Poner horario de llegada a la casa & 0.28 \\
\hline & & 173 Escuchar los problemas del hijo & 0.27 \\
\hline & & 172 Dar confianza & 0.21 \\
\hline & & 168 Establecer horario para hacer la tarea & -0.18 \\
\hline & \multirow{13}{*}{$\begin{array}{l}\text { Relación con la madre } \\
\text { de familia }\end{array}$} & 189 Escuchar los problemas del hijo & 0.71 \\
\hline & & 178 Reconocer el esfuerzo del hijo & 0.69 \\
\hline & & 179 Cuidar al hijo cuando está enfermo & 0.67 \\
\hline & & 185 Preguntar al hijo cómo le ha ido & 0.67 \\
\hline & & 188 Dar confianza & 0.67 \\
\hline & & 183 Encargarse de que estén alimentados & 0.63 \\
\hline & & 187 La mamá le platica al hijo lo que le sucede & 0.62 \\
\hline & & 190 Dar dinero para salidas & 0.46 \\
\hline & & 182 Sentirse bien cuando están juntos madre-hijo & 0.46 \\
\hline & & 177 Ponerse de acuerdo con su esposo & 0.34 \\
\hline & & 186 Asistir a las juntas de la escuela & 0.34 \\
\hline & & 191 Poner un horario de llegada a la casa & 0.30 \\
\hline & & Expl.Var & 7.50 \\
\hline \multicolumn{4}{|c|}{$-0.159 \geq r \geq 0.159$} \\
\hline$\overline{E j e}$ & Variable complejas & Variables simples & F3 \\
\hline $\begin{array}{l}\text { Aprendizaje } \\
\text { matemático }\end{array}$ & $\begin{array}{l}\text { Acciones que realiza } \\
\text { el estudiante en el } \\
\text { salón de clase } \\
\text { matemática }\end{array}$ & 9 Revisar programa & 0.23 \\
\hline \multirow{18}{*}{$\begin{array}{l}\text { Estilo de } \\
\text { vida }\end{array}$} & \multirow[t]{3}{*}{ Autopercepción } & 129 Estar a gusto con tu cuerpo & 0.29 \\
\hline & & 132 Sentirse satisfecho con la vida & 0.27 \\
\hline & & 126 Necesitar la aprobación de los demás & -0.22 \\
\hline & \multirow{3}{*}{$\begin{array}{l}\text { Acciones en tiempo } \\
\text { libre }\end{array}$} & 125 Fumar & -0.20 \\
\hline & & 131 Consumir alcohol & -0.20 \\
\hline & & 127 Enfermarse seguido & -0.37 \\
\hline & \multirow{8}{*}{$\begin{array}{l}\text { En tu alimentación } \\
\text { diaria sueles }\end{array}$} & 139 Verduras & 0.25 \\
\hline & & 144 Carne blanca & 0.23 \\
\hline & & 140 Carne roja & 0.21 \\
\hline & & 147 Frituras & -0.18 \\
\hline & & 142 Se salta comidas & -0.20 \\
\hline & & 138 Suplementos & -0.22 \\
\hline & & 141 Café & -0.28 \\
\hline & & 137 Se ingiera algo para adelgazar & -0.50 \\
\hline & \multirow{4}{*}{$\begin{array}{l}\text { Relación con los } \\
\text { amigos }\end{array}$} & 160 Tienen los mismos gustos & 0.20 \\
\hline & & 149 Jugar videojuegos & -0.27 \\
\hline & & 150 Sentirse solo sin amigos & -0.42 \\
\hline & & 153 Le hacen sentir excluido & -0.47 \\
\hline \multirow{10}{*}{$\begin{array}{l}\text { Habilidades } \\
\text { docentes }\end{array}$} & \multirow{4}{*}{$\begin{array}{l}\text { Manejo de la } \\
\text { tecnologia (por parte } \\
\text { del profesor) }\end{array}$} & 69 Blackboard & 0.29 \\
\hline & & 75 Software ejercicios (STU) & 0.20 \\
\hline & & 70 Proyectos en línea & 0.19 \\
\hline & & 72 Foros de discusión & -0.27 \\
\hline & \multirow{3}{*}{$\begin{array}{l}\text { Medición del } \\
\text { conocimiento }\end{array}$} & 78 Resolver examen individual & 0.45 \\
\hline & & 87 Consultar a 102 expertos del tema & -0.19 \\
\hline & & 83 Investigar en 203 revistas electrónicas & -0.20 \\
\hline & \multirow{3}{*}{$\begin{array}{l}\text { Acciones que realiza } \\
\text { el profesor en el salón } \\
\text { de clase matemática }\end{array}$} & 112 Estar de buen humor & 0.27 \\
\hline & & 96 Mostrar la planeación & 0.20 \\
\hline & & 108 Estimular la cooperación y trabajo en equipo & 0.19 \\
\hline
\end{tabular}

Tabla 8 Análisis Integracional: Factor 3 Alimentación saludable y contexto familiar como gestores de aprendizaje

Fuente: elaboración propia

$4^{\circ}$. Factor. Percepción matemática como condicionante del aprendizaje. Tiene un $12.39 \%$ de varianza y refiere a la importancia de el aprendizaje de las matemáticas en función de la percepción aprendida respecto a la materia.

JIMÉNEZ-HIDALGO, Guadalupe, MONTOYA-PONCE, Javier, MARTINEZ-ACOSTA, María Teresa y PEREA-SEAÑEZ, Loreley. Proyecto cultural para la adquisición de competencias laborales en Ingeniería Industrial. Revista de Didáctica Práctica. 2019 
La importancia de las acciones que realiza en clase y en casa para ayudar. Siendo también un factor significativo las habilidades docentes, las de gestión del aprendizaje la motivación a participar y la disposición de apoyo cuando tengan dudas de la asignatura.

La percepción positiva matemática gestiona el aprendizaje y se vincula con las acciones que realiza el estudiante en el aula como es ayudar a los compañeros, participar en equipo y pasar al pizarrón. También el estudiante no se queda con la duda y acude con el docente. Además, la actitud aprendida de matemáticas se relaciona con los procesos cognitivos para favorecer el aprendizaje y motivarse para que esto suceda.

\begin{tabular}{|c|c|c|c|}
\hline Eje & Variable compleja & Variable simple & Factor 4 \\
\hline \multirow{29}{*}{$\begin{array}{l}\text { Aprendizaje } \\
\text { matemático }\end{array}$} & \multirow{4}{*}{$\begin{array}{l}\text { Acciones que hace } \\
\text { el estudiante ante la } \\
\text { duda }\end{array}$} & 1 Maestro & 0.24 \\
\hline & & 7 Asistir asesorías & -0.25 \\
\hline & & 2 Tutor externo & -0.28 \\
\hline & & 6 Quedarse con la duda & -0.35 \\
\hline & \multirow{9}{*}{$\begin{array}{l}\text { Acciones que } \\
\text { realiza el estudiante } \\
\text { en el salón de clase } \\
\text { matemática }\end{array}$} & 27 Ayudar a compañeros & 0.35 \\
\hline & & 30 Proponer soluciones & 0.33 \\
\hline & & 21 Comprender instrucciones & 0.29 \\
\hline & & 13 Pensar cómo resolver & 0.23 \\
\hline & & 25 Participar activamente en equipo & 0.21 \\
\hline & & 15 Pasar al pizarrón & 0.21 \\
\hline & & 35 Evaluar opciones antes de resolver & 0.20 \\
\hline & & 19 Analizar ejercicio & 0.20 \\
\hline & & 14 Esperar que el profesor resuelva & -0.30 \\
\hline & \multirow{12}{*}{$\begin{array}{l}\text { Percepción de la } \\
\text { clase de } \\
\text { matemática }\end{array}$} & 46 La entiende & 0.70 \\
\hline & & 40 Es fácil & 0.70 \\
\hline & & 37 Le gusta & 0.69 \\
\hline & & 38 Lo hace sentir seguro & 0.69 \\
\hline & & 48 Es divertida & 0.55 \\
\hline & & 41 Se aplica a la vida diaria & 0.43 \\
\hline & & 47 Disfruta cómo imparte el profesor & 0.24 \\
\hline & & 42 Le es indiferente & -0.36 \\
\hline & & 43 Es aburrida & -0.54 \\
\hline & & 39 Lo pone nervioso & -0.58 \\
\hline & & 45 Lo derrota & -0.62 \\
\hline & & 44 Cuesta trabajo entenderla & -0.64 \\
\hline & \multirow{4}{*}{$\begin{array}{l}\text { Acciones que } \\
\text { realiza el estudiante } \\
\text { en casa con la tarea } \\
\text { matemática }\end{array}$} & 67 Reconoce ser responsable del aprendizaje & 0.25 \\
\hline & & 57 Invertir el tiempo hasta entender & 0.24 \\
\hline & & 49 Realizar ejercicios & 0.23 \\
\hline & & 60 Tener beca & 0.22 \\
\hline \multirow[t]{2}{*}{$\begin{array}{l}\text { Habilidades } \\
\text { docentes }\end{array}$} & $\begin{array}{l}\text { Acciones que } \\
\text { realiza el profesor } \\
\text { en el salón de clase } \\
\text { matemática }\end{array}$ & 93 Motivar a participar & 0.16 \\
\hline & & Expl.Var & 5.94 \\
\hline
\end{tabular}

Tabla 9 Análisis integracional Factor 4: Percepción como condicionante del aprendizaje matemático.

Por lo tanto, la percepción positiva, las acciones escolares áulicas y en casa, la utilización de las habilidades cognitivas son el locus de control interno que posibilitan el aprendizaje, además las habilidades docentes son el apoyo externo.

Loa demás factores presentes son:

$5^{\circ}$. Autopercepción acorde al roll de la figura paterna como base del aprendizaje matemático. $6^{\circ}$ Percepción matemática acorde a acciones dlel estudiante. de iguales.

$7^{\circ}$. Aprendizaje matemático como apoyo

$8^{\circ}$. Aprendizaje matemático acorde al estilo de vida saludable y autopercepción.

$9^{\circ}$. Atributos externos que se asocian al aprendizaje matemático.

$10^{\circ}$ Atributos del estilo de vida.

$11^{\circ}$ Los amigos apoyo del aprendizaje matemático.

$12^{\circ}$ Aprendizaje matemático acorde al reglamento familiar.

$13^{\circ}$ Organización familiar del tiempo. adolescente.

$14^{\circ}$ Atributos negativos propios del

$15^{\circ}$ Acciones de casa para aprender matemáticas.

$16^{\circ}$ Creatividad docente.

$17^{\circ}$ Didáctica docente acorde al ambiente familiar.

$18^{\circ}$ Atributos que dificultan el aprendizaje matemático.

$19^{\circ}$ Conductas negativas que dificultan el aprendizaje matemático.

$20^{\circ}$ Aprendizaje matemático centrado en acciones escolares,

\section{Conclusiones}

El aprendizaje del ser humano, en las abstracciones necesita orientación que haga que las matemáticas contribuyan a su desarrollo. Por ello en ésta investigación podemos constatar que la autopercepción origina en el estudiante el desarrollo hacia un estilo de vida saludable, con relaciones positivas entre compañeros, además desarrollo de habilidades cognitivas empoderan la autonomía para lograr ser gestor de su propio aprendizaje. 
Los atributos que se asocian en el aprendizaje; como la autopercepción vinculada al autoaprendizaje, la autonomía con la adquisición de conocimiento, se puede constatar estadísticamente una disminución en el aprendizaje centrado en el docente, asumiendo el estudiante el rol de responsabilidad del aprendizaje hacia sí mismo, al desarrollar habilidades de la forma en que aprende, en cómo analiza, evalúa, comprende, planea y propone soluciones para resolver un problema. Así como también, el reconocerse como responsable de las acciones concretas en el aula, que lo empoderan como agente activo del aprendizaje. Entre ellas están: el pasar al pizarrón en clases, participar activamente en equipos de trabajo y apoyar a los compañeros cuando tienen dificultades para resolver problemas. Por último, la investigación muestra las vinculaciones significativas que resultan de las relaciones familiares en especial con el padre cuando le otorga confianza y mantiene una buena comunicación al escuchar al hijo y muestra estar al pendiente de lo que sucede, todo eso propicia el ambiente idóneo que favorece al aprendizaje.

En síntesis, se hacen presentes los actores educativos de los modelos educativos propuestos en teorías del aprendizaje tradicionales de la enseñanza orientada en el docente, al mismo tiempo se reflejan las innovaciones actuales del aprendizaje centrado en el estudiante bajo el modelo de competencias. La investigación muestra la objetividad de factores que son determinantes en alguna forma pero a veces no mesurables y apreciados en teorías pedagógicas, existen factores que potencializan o limitan cualquier aprendizaje humano, estos otros atributos refieren a características que les facilitan a los estudiantes el aprender matemáticas, como las conductas presentadas ante dudas al resolver problemas abstractos, reconocer la necesidad de la experiencia guía del pedagogo, ejercitar habilidades cognitivas, practicar en clase, apreciación de actitudes positivas con acento emocional hacia estos temas, actividades a realizar en casa como tareas de ejercicios, tiempo dedicado al estudio, autorresponsabilidad al aprender y el percibir de forma lúdica, como diversión el aprendizaje en matemáticas.
Cuando los estudiantes tienen cuestionamientos de matemáticas acuden, por prioridades primero al maestro, a los amigos, a los compañeros de clase o investigan para aclarar las dudas y completar el aprendizaje recurriendo a fuentes externas como los tutores. Desde la perspectiva de los estudiantes, la importancia de la relación con amigos tiene variables que potencializan el aprendizaje matemático como: platicar vía internet, sentirse aceptado, tener gustos similares y jugar videojuegos en tiempo libre a los hombres y a las mujeres les gusta platicar en forma presencial entre pares.

Los estudiantes opinan que la alimentación es saludable y balanceada, probablemente se deba al nivel de vida al que pertenecen, ya que la población encuestada tiene estatus económico medio-alto, pues los estudiantes asisten a preparatorias del sector privado, donde la colegiatura semestral por cada estudiante equivale aproximadamente a 26 salarios mínimos en el estado de Nuevo León.

De la autopercepción las variables significativas para el apoyo al aprendizaje matemático están: sentirse satisfecho con la vida, estar a gusto con el físico y estar de buen humor. Los atributos negativos presentes complementan a los positivos anteriormente, por ejemplo, cuando tiene duda no se queda sin resolverlas pues acude al maestro, amigos o compañeros. Los estudiantes no están siempre a expensas de los maestros pues van y aclaran las dudas en fuentes externas. Algunos estudiantes que se correlacionan con hábitos de consumo de bebidas alcohólicas y fuman, no siguen instrucciones de los pedagogos, no utilizan agenda, no leen el libro. La significancia negativa se presenta en atributos de los estudiantes que son autónomos como: el no ponerles nerviosa la clase de matemáticas, no les es indiferente, no les cuesta trabajo entenderla, ni les es aburrida. El aprendizaje de las matemáticas puede facilitarse $y$ potencializarse con la intervención de aspectos que refieren a los estudiantes y el comportamiento dentro y fuera del aula, en donde el maestro es una figura importante $y$ necesitada por ellos, además de aspectos, cognitivos, actitudinales y emocionales, que se relacionan con amigos y relaciones parentales, así como factores de hábitos de estudio, salud y autoestima. 
El aprendizaje matemático es un fenómeno complejo de participación multifactorial y multidireccional que incluye diferentes y variados aspectos que se conjugan en proporciones muchas veces no prospectadas del intelecto humano, por lo que es importante seguir investigando metodológicamente para innovar con nuevas aportaciones al proceso enseñanza-aprendizaje en matemáticas, donde a esa edad se tiene mayor dificultad para asimilarla y aplicarla a la vida real.

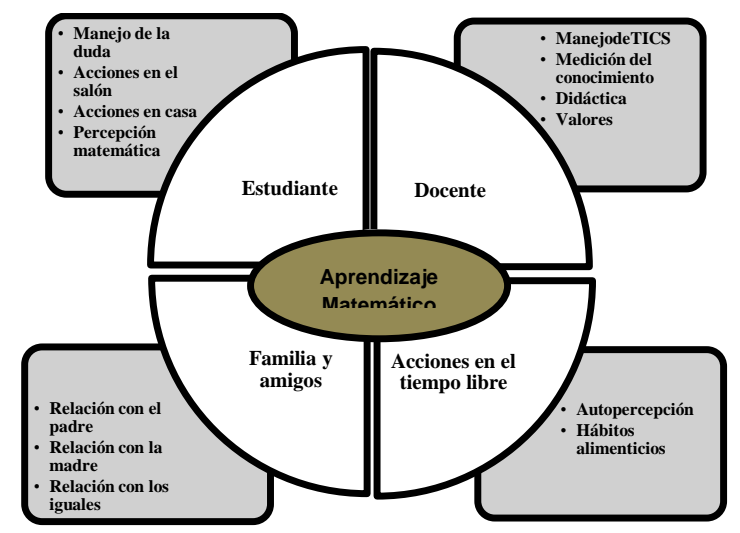

Figura 1

\section{Propuestas de transformación}

Los atributos del proceso de aprendizaje matemático, el interés por investigar dichos constructos surge de la necesidad de detectar los elementos no contemplados con anterioridad en la didáctica de los números, para tenerlos en consideración e integrarlos al modelo educativo actual en nivel de educación media superior.

Al identificar los atributos del contexto familiar, los hábitos de estudio y apoyos parentales que se vinculan con la forma en cómo aprenden los estudiantes de nivel medio superior. Se elaborarán programas de orientación a padres de familia y capacitación a docentes para realizar acciones de transformación educativas y pedagógicas como: diseñar una propuesta curricular integral nueva basada en las necesidades reales del adolescente, incidir en la praxis docente, para integrar en programas de actividades curriculares los factores que se asocien al aprendizaje de los estudiantes.
Además, se puede diseñar un cuestionario como instrumento de diagnóstico inicial para preparatoria, para tener un informe diagnóstico de habilidades, destrezas, hábitos y organización familiar que puede apoyar al aprendizaje de los estudiantes, con información a priori se pueden diseñar los programas y actividades didáctico matéticas personalizadas a estudiantes para visualizar oportunidades de desarrollo o remediar en su caso deficiencias que se presenten. Asumiendo que los docentes son parte de la solución para mejorar el rendimiento académico, se les sensibilizará y concientizará a los docentes de las herramientas pedagógicas, disciplinares y psicológicas pertinentes para identificar y mejorar el aprendizaje de acuerdo a las necesidades de los estudiantes. Otra aportación es preparar a los estudiantes las aptitudes que les ayuden a desenvolverse en el mundo del trabajo o para incursionar en la educación superior y cultivar en ellos las habilidades imprescindibles para desempeñarse de manera competitiva en la sociedad moderna.

\section{Referencias}

Abello, A. y Montaño, J. (2013). Leer y comprender para aprender matemática. VARONA, Revista Científico-Metodológica. 1(57), 60-68. Recuperado Noviembre, 23, 2016 de

http://www.redalyc.org/articulo.oa?id=3606341 64012

Andere, E. (2007). ¿Cómo es la mejor educación en el mundo? Políticas educativas y escuelas en 19 países. México: Editorial Santillana, S.A.

Bar, G. (1999). Perfil y competencias del docente en el contexto institucional educativo. Organización para los Estados Iberoamericanos para la Educación, la Ciencia y la Cultura. Primer Seminario Taller sobre Perfil del Docente y Estrategias de Formación. Recuperado Diciembre, 03, 2016 de http://server1.docfoc.com/uploads/Z2016/01/09 /V5Wy5DdfIC/c937541c97335cbb54767beaf6 2ff55e.pdf

Bassanezi, R. C. y Biembengut, M. S. (1997). Modelación matemática: Una antigua forma de investigación - Un nuevo método de enseñanza. Revista didáctica de las matemáticas. 2(1), 1325 .

JIMÉNEZ-HIDALGO, Guadalupe, MONTOYA-PONCE, Javier, MARTINEZ-ACOSTA, María Teresa y PEREA-SEAÑEZ, Loreley. Proyecto cultural para la adquisición de competencias laborales en Ingeniería Industrial. Revista de Didáctica Práctica. 2019 
Bastias, M. y Bertoni, J. (2014). Una revisión de los estilos de vida de estudiantes universitarios Iberoamericanos. Revista Ciencia y Enfermería XX. (2), 93-101. Recuperado Diciembre, $\quad 03, \quad 2016 \quad$ de http://www.scielo.cl/pdf/cienf/v20n2/art_10.pdf

Bishop, A. (1991). Mathematical Enculturation: A Cultural Perspective on Matetmatics. Dordrencht: Kluwer Academic Publisher. Recuperado Marzo 02, 2018 de: http://www.springer.com/gp/book/9780792312 703

Bohorquez, L. (2014). Las creencias vs. Las concepciones de los profesores de matemáticas y sus cambios. Congreso Iberoamericano de Ciencias, Tecnología, Innovación y Educación. Artículo 1611, 1-27. Recuperado Mayo 27, 2018 de: http://www.google.com.mx/url?sa=t\&rct=j\&q= $\&$ esrc $=$ s\&source $=$ web $\& c d=2 \& v e d=0$ ahUKEwi D6uSlkafbAhUPYK0KHSHECIIQFggvMAE\& url=http $\% 3 \mathrm{~A} \% 2 \mathrm{~F} \% 2 \mathrm{Fwww}$. oei.es\%2Fhistorico \%2Fcongreso2014\%2Fmemoriactei\%2F1611

Camarena P. (2014). La matemática social en el desarrollo integral del alumno. Revista: Innovación Educativa. 14 (65), núm. 65,143149. Recuperado Noviembre, 23, 2016 de http://www.redalyc.org/articulo.oa?id=1794315 12010

Campos, Y. (2001). Enfoque humanista de la educción matemática y elementos efectivos de su enseñanza. Recuperado Mayo 26, 2018 de http://www.camposc.net/0repositorio/ponencias /01humanista.pdf

Cantoral, R., Farfán, R., Alanís, J., Rodríguez, R. y Garza, A. (2000). Desarrollo del pensamiento matemático, México. Ed. Trillas.

Cantoral, R. y Hernández, D. (2013). Teoría socioepistemológica de la matemática educativa: Estudio sobre la construcción social del conocimiento. México.: Gedisa

Carvallo, M. (2005). Análisis de resultados obtenidos en estudios de eficacia escolar en México, comparados con los de otros países. REICE. Revista Iberoamericana sobre Calidad, Eficacia y Cambio en Educación. 3(2), 80-108. Recuperado Noviembre, 23, 2016 de http://www.redalyc.org/articulo.oa?id=5510320 7
Carvallo, M. (2006). Factores que Afectan el Desempeño de los Alumnos Mexicanos en Edad de Educación Secundaria: Un Estudio Dentro de la Corriente de Eficacia. REICE. Revista Iberoamericana sobre Calidad, Eficacia y Cambio en Educación. 4(3), 30-53. Recuperado Noviembre, 23, 2016 de http://www.redalyc.org/pdf/551/55140304.pdf

Cedillo, T. (2006). La enseñanza matemática en la escuela secundaria. Revista Mexicana de Investigación Educativa. 11(28), 129-153. Recuperado Noviembre, 23, 2016 de http://www.redalyc.org/articulo.oa?id=1400280 7

Cervini, R. (2004). Influencia de los factores institucionales sobre el logro en las matemáticas de los estudiantes en el último año de Educación Media de Argentina. Un modelo de tres niveles. Revista electrónica Iberoamericana sobre Calidad, Eficacia y Cambio en la Educación 2(1), 1-24. Recuperado Noviembre, 23, 2016 de http://www.ice.deusto.es/RINACE/reice/vol2n1 /Res_Cervini.htm

D’Ambrosio, U. (1985). Socio-cultural bases for mathematics education. Campinas.: UNICAMP.

Delors, J. (1996). La educación encierra un tesoro. Informe a la UNESCO de la Comisión Internacional sobre la educación para el siglo $X X I$. Ediciones UNESCO. : España.

Fernández, R., Suárez J. y Muñiz J. (2016). Deberes y rendimiento en matemáticas: papel del profesorado, la familia y las características del alumnado. Revista de Psicodidáctica DIALNET. 21(1), 5-23. Recuperado Noviembre, 23, 2016 de https://dialnet.unirioja.es/servlet/autor?codigo= 22791http://www.ehu.eus/ojs/index.php/psicodi dactica/article/view/13939/13521

Flores S., Ángel H., Gómez A. (2009). Aprender Matemática, Haciendo Matemática: la evaluación en el aula. Revista Educación Matemática. 21(2), 117-142 Recuperado Diciembre, $\quad 03, \quad 2016 \quad$ de http://www.redalyc.org/articulo.oa?id=4051667 2005 